\title{
Educar para a Saúde Laboral: Perceção da Qualidade de Vida em relação a variáveis sociodemográficas, condições de Saúde e de Trabalho em trabalhadores de escritório
}

rpso.pt/educar-para-a-saude-laboral-percecao-da-qualidade-de-vida-em-relacao-a-variaveis-sociodemograficascondicoes-de-saude-e-de-trabalho-em-trabalhadores-de-escritorio/

Moreira S, Miranda A, Lima C, Gonçalves C, Mesquita C, Lopes S, Machado J, Santos P. Educar para a Saúde Laboral: Perceção da Qualidade de Vida em relação a variáveis sociodemográficas, condições de Saúde e de Trabalho em trabalhadores de escritório. Revista Portuguesa de Saúde Ocupacional on line. 2020, volume 10, 1-19. DOI: 10. 31252/RPSO.19.12.2020

EDUCATING FOR OCCUPATIONAL HEALTH: PERCEPTION OF QUALITY OF LIFE IN RELATION TO SOCIO-DEMOGRAPHIC VARIABLES, HEALTH AND WORK CONDITIONS IN COMPUTER WORKERS

TIPO DE ESTUDO: Observacional Analítico Transversal

Autores: Moreira S(1), Miranda A(2), Lima C(3), Gonçalves C(4), Mesquita C(5), Lopes S(6), Machado J(7), Santos P(8).

\section{RESUMO}

\section{Introdução}

Os trabalhadores de escritório permanecem em posições estacionárias durante longos períodos e estão sujeitos a elevadas cargas laborais que podem interferir com a Qualidade de Vida, em interação com caraterísticas do trabalhador (como personalidade, autonomia, competência e empenho no trabalho). A Qualidade de Vida é um conceito multidimensional influenciado por fatores sociodemográficos, de saúde e trabalho, que se tornou um desafio de Saúde Ocupacional, pela preocupação crescente das organizações que atuam a esse nível.

\section{Objetivos}

Avaliar a perceção da Qualidade de Vida e relacioná-la com variáveis sociodemográficas, condições de saúde e de trabalho.

\section{Métodos}


Trata-se de um estudo observacional analítico transversal, com uma amostra de 119 trabalhadores de escritório (sendo 82 indivíduos do género masculino) de uma empresa fabricante de componentes automóveis. Utilizou-se o questionário SF-36v2 com o objetivo de avaliar a perceção da Qualidade de Vida relacionada com a saúde, bem como um questionário de caracterização da amostra para recolha de dados sociodemográficos, de saúde e trabalho, preenchidos no GoogleForms. Na análise dos dados foi realizada estatística descritiva e inferencial, com nível de significância 0,05, através do IBM SPSS Statistics versão 25.0.

\section{Resultados}

Os trabalhadores de escritório apresentaram em todas as dimensões da Qualidade de Vida pontuações acima de 52 em 100. Os itens físicos foram os que apresentaram maiores pontuações sendo a dimensão Função Física a que apresentou maior pontuação $(95,3 \pm 7,74)$, as dimensões mentais apresentaram pontuações menores, nomeadamente o Desempenho Emocional $(52,1 \pm 43,10)$ e a Vitalidade $(57,9 \pm 24,16)$.

Relativamente à análise das variáveis sociodemográficas e Qualidade de Vida, o género masculino apresentou uma melhor perceção da Qualidade de Vida com diferenças estatisticamente significativas nas dimensões Vitalidade $(p=0,047)$ e Dor $(p=0,043)$.

Quanto às condições de saúde, verificou-se que a variável Obesidade apresentou diferenças estatisticamente significativas nas dimensões Função Física $(p=0,001)$ e Estado de Saúde Geral ( $p=0,003)$, sendo os trabalhadores com obesidade os que apresentaram maior afetação nestas dimensões.

Ser portador de doença crónica influenciou negativamente as dimensões Estado de Saúde Geral $(p=0,034)$ e Vitalidade $(p=0,047)$. Assim como estar de baixa médica afetou as dimensões Função Social $(p=0,019)$ e Saúde Mental $(p=0,001)$.

Relativamente às condições de trabalho, verificaram-se diferenças significativas entre a variável Função de Direção e a dimensão Desempenho Físico $(p=0,001)$, sendo os trabalhadores que exercem funções de direção os que apresentam valores mais baixos nesta dimensão.

\section{Conclusão}

A perceção da Qualidade de Vida diferiu nos diferentes níveis considerados, tendo-se verificado que as dimensões mentais foram as mais afetadas (Desempenho Emocional e Vitalidade); por outro lado, as dimensões físicas foram as que mais contribuíram para a boa perceção da Qualidade de Vida dos trabalhadores. As ofertas implementadas pela empresa podem ter influenciado positivamente os resultados do estudo e justificar a boa perceção global da Qualidade de Vida destes trabalhadores de escritório.

Palavras-chave: Saúde Ocupacional, SF-36v2, Trabalhadores de Computador 


\section{ABSTRACT}

\section{Background}

Computer workers remain in the stationary positions for long periods and are subject to high workloads that can interfere with Quality of Life, taking into account the worker's characteristics (such as personality, autonomy, competence and commitment to work). Quality of Life is a multidimensional concept influenced by socio-demographic, health and work factors, which has become an occupational health challenge, due to the growing concern of organizations working at this level.

\section{$\operatorname{Aim}(\mathbf{s})$}

To evaluate the perception of Quality of Life and relate it to sociodemographic variables, health and work conditions.

\section{Methods}

This is a cross-sectional observational analytical study, with a sample of 119 office workers (82 male and 37 female) from an automotive components manufacturer. The SF-36v2 questionnaire was used to evaluate the perception of Quality of Life related to health, and a sample characterization questionnaire to collect socio-demographic, health and work data, filled out on Google Forms. In the data analysis, descriptive and inferential statistics, with a significance level of 0.05 , were performed through IBM SPSS Statistics version 25.0 .

\section{Results}

Office workers scored in all dimensions of Quality of Life scores above 52 out of 100 . The physical dimensions were the ones that presented the highest scores, being the Physical Function dimension the one with the highest score $(95.3 \pm 7.74)$, the mental dimensions presented lower scores, namely Emotional Performance $(52.1 \pm 43.10)$ and Vitality (57.9 \pm 24.16$)$.

Regarding the analysis of socio-demographic variables and Quality of Life, male gender presented a better perception of Quality of Life with statistically significant differences in Vitality $(p=0.047)$ and Pain $(p=0.043)$ dimensions.

Regarding health conditions, it was verified that the variable Obesity presented statistically significant differences in the dimensions Physical Function $(p=0.001)$ and General Health Status $(p=0.003)$, being the workers with obesity the most affected in these dimensions.

Being chronically ill negatively influenced the dimensions General Health Status $(p=0.034)$ and Vitality $(p=0.047)$. As well as being on sick leave affected the dimensions Social Function $(p=0.019)$ and Mental Health $(p=0.001)$. 
Regarding the working conditions, there were significant differences in the variable steering function and the Physical Performance dimension $(p=0.001)$, being the workers with steering functions the ones with lower values in this dimension.

\section{Conclusion}

The perception of the Quality of Life differed in the different dimensions, having been verified that the mental dimensions were the most affected (Emotional Performance and Vitality), on the other hand the physical dimensions were the ones that most contributed to the good perception of the Quality of Life of the workers. The offers implemented by the company may have positively influenced the results of the study and justify a good overall perception of the Quality of Life of these office workers.

Key words: Occupational Health, SF-36v2, Computer Workers

\section{INTRODUÇÃO}

A Organização Mundial de Saúde (OMS) (1) define a Qualidade de Vida (QV) como a perceção do indivíduo sobre a sua posição na vida, dentro do contexto dos sistemas de cultura e valores nos quais está inserido e em relação aos seus objetivos, expectativas, padrões e preocupações.

Segundo a OMS (1), a QV é multidimensional, engloba diversos aspetos do quotidiano do indivíduo numa abordagem biopsicossocial, nomeadamente a nível físico, psicológico e social (1-3). Este conceito é subjetivo e tende a mudar ao longo do tempo. Estas diferenças verificam-se entre géneros, classes etárias, nível educacional, presença ou ausência de fatores adversos, profissões, estilos de vida, entre outros aspetos, que afetam a perceção dos indivíduos sobre o seu estado de saúde $(1,4-7)$.

O conceito de saúde pode ser visto e interpretado numa perspetiva individual, social e cultural (8). De acordo com a OMS (1), a saúde é vista como um recurso para a vida de todos os dias, sendo uma das dimensões da QV $(1,7,9)$. Medir o estado de saúde das populações permite definir níveis de comparação entre grupos acerca das condições de saúde (por diferentes patologias), condições de trabalho (como horas de trabalho, situação de trabalho, funções de chefia), condições sociais, condições económicas ou ainda relativas ao género e à idade (9).

O bem-estar físico, mental, económico e social dos trabalhadores é influenciado pelo contexto de trabalho que, segundo a Direção Geral da Saúde (2), quando seguro e saudável, terá inevitavelmente reflexo positivo na sua saúde e bem-estar bem como das suas famílias, comunidades e sociedade em geral. 
Com a crescente afluência à tecnologia, os postos de trabalho implicam a repetição constante de movimentos e a adoção de postura sentada mantida durante grande parte da jornada laboral, promovendo comportamentos sedentários que inerentemente afetam a QV $(10,11)$.

Os trabalhadores de escritório, enquadram-se neste grupo, pois permanecem a maior parte do tempo neste conjunto postural, executando tarefas variadas. Este comportamento, sem pausas de descanso leva a uma utilização excessiva de sistemas, nomeadamente neuromusculoesquelético, o que pode provocar desconforto, fadiga ou trauma $(10,12-16)$.

Existem fatores associados ao trabalho que influenciam a QV dos trabalhadores, como uma alta carga mental, prazos curtos para entrega dos trabalhos, alto grau de responsabilidade e intensa exposição ao computador $(12,13,17,19)$. Segundo a European Agency for Safety and Health at Work (20), a frequência desses fatores está associada a níveis mais elevados de ansiedade, problemas de sono, fadiga geral e diminuição do bem-estar mental dos trabalhadores. Esses fatores de risco associam-se à forma como o trabalho é realizado, organizado e gerido, bem como ao contexto económico e social, originando uma deterioração da saúde mental e física, fatores determinantes para a QV do trabalhador $(8,17,18)$.

A melhoria da QV é um desafio de saúde ocupacional e tal como refere a OMS (1), o contexto de trabalho é fundamental para o desenvolvimento e criação de ações de promoção de saúde no âmbito da prática de trabalho e de estilo de vida saudável (21).

Após verificação da literatura referente à Saúde Ocupacional observou-se que tem vindo a crescer a preocupação com a saúde do trabalhador. Assim, este estudo teve por objetivo avaliar a perceção da QV dos trabalhadores de escritório de uma empresa fabricante de componentes automóveis e verificar se existe relação com as variáveis sociodemográficas, condições de saúde e condições de trabalho.

\section{METODOLOGIA}

\section{Desenho de estudo}

Realizou-se um estudo observacional analítico transversal.

\section{Amostra}

A população alvo do estudo são os trabalhadores de escritório da empresa BorgWarner de Viana do Castelo. A seleção da amostra foi não probabilística, por conveniência.

Como descrito na Figura 1, para a seleção da amostra foram estabelecidos critérios de inclusão, nomeadamente, ter idade compreendida entre 18 e 65 anos. E, como critérios de exclusão serem portadores de patologias genéticas, reumáticas ou neurológicas e 
terem algum tratamento fora do contexto de trabalho (sessões de fisioterapia ou psicologia, por exemplo). Do total de 424 trabalhadores, 120 participaram voluntariamente. Foi excluído um participante, por ter o diagnóstico de espondilite anquilosante. Sendo a amostra final de 119 participantes, correspondendo a $28,3 \%$ da população.

Realizou-se o cálculo do tamanho da amostra através do software $\mathrm{R}$, de modo a verificar a sua representatividade relativamente à população alvo. A amostra é representativa tendo como pressupostos um nível de significância de 5\% a duas caudas (ou seja, um nível de confiança de 95\%) e uma precisão de 7,6\%.

\section{Instrumentos}

Os instrumentos utilizados neste estudo foram o Questionário de Caraterização da amostra e o Questionário de Avaliação da Qualidade de Vida Relacionada com a Saúde (SF-36v2) (22).

\section{Questionário de Caraterização da amostra}

Este questionário foi adaptado para o presente estudo, pelo que foi dividido em três partes. A primeira, relacionada com caraterísticas sociodemográficas, permite caracterizar a amostra através da recolha de dados como o Género, Idade e Habilitações Literárias (agruparam-se as respostas em possuir ensino superior - Bacharelato, Licenciatura, Mestrado e Doutoramento-, ou não possuir ensino superior $-2^{\circ}, 3^{\circ}$ ciclo, Ensino secundário e Curso profissional + CET). A segunda, relacionada com as condições de saúde dos indivíduos da amostra, possibilitou recolher informações sobre altura e peso que permitiu o cálculo do IMC (Índice de Massa Corporal) e a categorização (segundo os valores da OMS) da amostra em obesos ( $\geq 30 \mathrm{~kg} / \mathrm{m}^{2}$ ) e não obesos (<29,9 kg/m²), Doença Crónica (através da pergunta "É portador de alguma doença diagnosticada?") e Certificado de Incapacidade Temporária ("Baixa Médica") no último ano. A terceira parte deste questionário está direcionada para questões relacionadas com as condições de trabalho, nomeadamente o número de horas extraordinárias e extralaborais (realizadas no trabalho e em casa), se exerce funções de direção (cargos intermédios de direção, chefia ou coordenação, que exigem maior responsabilidade), e a situação atual de emprego (pertencer ou não ao quadro da empresa).

\section{Questionário de Avaliação da Qualidade de Vida Relacionada com a Saúde (SF- 36v2)}

O questionário SF-36v2 tem como finalidade avaliar a perceção da QV relacionada com a saúde de populações e indivíduos com ou sem doença (22). É de autopreenchimento e tem a duração aproximada de dez minutos. Constituído por 36 itens, distribuídos por oito dimensões, cada uma pontuada de 0 a 100 (quanto mais baixa a pontuação, menor a perceção da QV do indivíduo) (22). 
As diferentes áreas do questionário permitem avaliar oito dimensões do estado de saúde (22). A Função Física destina-se a avaliar o impacto na QV das limitações físicas. O Desempenho Físico e Emocional mede o impacto das limitações em saúde devido a problemas físicos ou emocionais (22). A Dor avalia a intensidade e o desconforto originados pela dor e de que modo é que esta interfere com o trabalho normal; o Estado de Saúde Geral avalia a perceção holística da saúde (22). A dimensão da Vitalidade, engloba os níveis de energia e de fadiga, enquanto a Saúde Mental inclui conceitos de ansiedade e depressão (22). Por fim, a Função Social, compreende a quantidade e a qualidade das atividades sociais e o impacto dos problemas físicos e emocionais nestas atividades $(7,22)$. Podem agrupar-se em duas dimensões gerais de estado de saúde:

física e mental. A dimensão física compreende a Função Física, o Desempenho Físico, a Dor e o Estado de Saúde Geral e a dimensão mental é constituída pelas restantes $(22,23)$.

O SF-36v2 encontra-se traduzido e validado para a população portuguesa e apresenta uma consistência interna muito boa, com valores de alfa de Cronbach compreendidos entre 0,6 na Função Social e 0,9 para Função Física e Estado Saúde Geral (22).

\section{Procedimentos}

Foi realizada uma visita à empresa para observar as condições de trabalho. Os questionários foram inseridos na plataforma Google Forms para serem preenchidos facilmente e rapidamente por todos os trabalhadores de escritório, o link para o preenchimento foi enviado por e-mail pelo departamento dos Recursos Humanos, para garantir o anonimato dos trabalhadores. O questionário esteve disponível para preenchimento entre o dia 5 e 12 de junho de 2019. As respostas aos questionários foram exportadas para o Excel. De forma a facilitar a leitura dos dados, foi realizada uma ponderação de dados de modo a atribuir as devidas pontuações a cada item, após isso foi realizado o cálculo do Raw Scale, convertendo as pontuações obtidas no SF-36v2 para uma escala de 0 (pior) a 100 (melhor) QV (24).

Posteriormente os dados foram importados para o programa IBM SPSS Statistics versão 25.0 (Statistical Package for the Social Sciences®, IBM Lisboa, Portugal) para posterior análise estatística.

\section{Ética}

O estudo foi aprovado pela Comissão de Ética do CHUP/ICBAS ( $n^{\circ}$ de referência 963), e foi admitido um acordo de confidencialidade com a administração da empresa BorgWarner. Todos os participantes consentiram e foram informados acerca do objetivo e da pertinência do estudo, assim como foi assegurada a proteção dos dados recolhidos e o seu anonimato. Os resultados foram armazenados numa base de dados, à qual apenas os investigadores tiveram acesso, e só foram utilizados para divulgação científica. 
Os participantes puderam rejeitar ou suspender a participação no estudo a qualquer instante de acordo com a lei 67/98 de outubro e Declaração de Helsínquia da Associação Médica Mundial.

\section{Estatística}

A análise estatística foi realizada através do programa IBM SPSS Statistics versão 25.0, com o intuito de realizar a estatística descritiva e inferencial, com um nível de significância de 0,05.

A estatística descritiva foi realizada através de medidas de tendência central, (nomeadamente médias), medidas de dispersão (desvio-padrão, máximo e mínimo) e frequências absolutas e relativas, para caracterizar a amostra.

A estatística inferencial foi realizada através de testes de hipóteses, consoante a relação das diferentes variáveis. Os testes Shapiro Wilk $(n<50)$ e Kolmogorov-Smirnov $(n>50)$ indicaram se os dados seguiam uma distribuição normal, ou não. Quando se identificou normalidade dos dados realizou-se o teste paramétrico $T$ para amostras independentes caso contrário foram utilizados testes não paramétricos, nomeadamente Mann-Whitney e Kruskal-Wallis. Para analisar a relação entre as variáveis procedeu-se a cálculos de regressão linear simples e foi utilizado o coeficiente de determinação e de correlação de Spearman, considerando-se uma correlação muito forte quando o valor estava entre 0.91 , forte entre 0.6-0.9, moderada entre 0.3-0.6, e fraca entre 0-0.3 (25).

\section{RESULTADOS}

\section{Caraterização da amostra}

A Tabela 1 mostra as características da amostra em estudo. Observou-se que esta é constituída por 119 indivíduos, sendo que 82 são do género masculino. Os trabalhadores de escritório apresentaram uma média de idade de 35,8 \pm 6,88 anos e apenas 2,9\% têm idade superior a 50 anos. Relativamente às Habilitações literárias, 56,3\% concluiu um curso superior, verificando-se uma diferença estatisticamente significativa entre o género feminino e masculino $(78,4 \%$ vs. $46,3 \%$, respetivamente).

Dentro das variáveis relativas à condição de saúde, descritas na Tabela 1, observou-se que a amostra apresenta uma média de IMC de $25,2 \pm 4,21 \mathrm{~kg} / \mathrm{m}^{2}$, sendo que o género masculino apresenta uma média superior. Verificou-se que $15,1 \%$ da amostra encontrase na categoria obesa, com o género masculino dominante (17,1 vs. 10,8, respetivamente). Relativamente à Doença Crónica, $12,6 \%$ do total da amostra referiu ser portadora, sendo esta mais frequente no género feminino (16,2\% vs. 11,0\%). Estes referiram as seguintes patologias: tumor, asma, psoríase, otosclerose auditiva, hérnia 
discal, bronquite crónica, hipertensão arterial e hipotiroidismo. Ainda dentro das variáveis relativas às condições de saúde, verificou-se que $6,7 \%$ do total da amostra esteve de Baixa Médica no ano anterior a responder ao questionário.

Relativamente às condições de trabalho, referidas na Tabela $1,74,0 \%$ da amostra faz Horas extraordinárias, e dentro dessa população, $75,7 \%$ é do género feminino; contatouse que, em média, os trabalhadores de escritório trabalham mais 5,3 $\pm 5,08$ horas. Verificou-se ainda que 40,3\% da amostra exerce Funções de direção, sendo que 46,3\% do género masculino e $27,0 \%$ do género feminino assumem esse cargo. Por fim, na Situação atual de emprego, verificou-se que 83,2\% dos participantes pertencem ao quadro da empresa, sendo que o género masculino se apresenta em maioria com uma diferença estatisticamente significativa; o número de trabalhadores de escritório do género masculino que pertence ao quadro é $18,7 \%$ superior ao feminino.

\section{Perceção da QV e variáveis sociodemográficas, condições de saúde e condições de trabalho}

Pela análise das Tabelas 2 e 3, verificou-se que a amostra total apresentou em todas as dimensões da QV pontuações acima de 50, sendo a Função Física a que apresentou maior pontuação $(95,3 \pm 7,74)$, e menor pontuação o Desempenho Emocional $(52,1 \pm 43,10)$, seguido de Desempenho Físico $(54,8 \pm 40,03)$ e Vitalidade $(57,9 \pm 24,16)$. De uma forma global, verificou-se que as dimensões mentais (exceto a Função Social) apresentaram médias de pontuação da QV menores relativamente às dimensões físicas. Importante referir que algumas dimensões da QV apresentaram valores elevados de desvio padrão nomeadamente o Desempenho Emocional e o Desempenho Físico.

Nas variáveis sociodemográficas, observaram-se diferenças significativas entre o género feminino e o masculino e as dimensões Dor e Vitalidade (69,8 vs. 78,8 e 51,4 vs. 60,9 respetivamente). As mulheres apresentaram tendencialmente valores mais baixos em todas as dimensões da QV (exceto no Estado de Saúde Geral). Por sua vez, na variável Habilitações literárias, não se observou nenhuma diferença estatisticamente significativa, no entanto, é de referir que os indivíduos que possuem ensino superior, apresentaram todos pontuações ligeiramente superiores relativamente aos restantes, à exceção da Dor.

Relativamente às condições de saúde, verificou-se que a variável Obesidade apresentou diferenças estatisticamente significativas, nas dimensões Função Física (apesar desta dimensão ser minimamente afetada na perceção de QV pelos trabalhadores de escritório, pois apresentou valores acima de 91,9) e Estado de Saúde Geral, sendo que os trabalhadores de escritório obesos, apresentaram estas dimensões mais afetadas. Para além disso, apesar de não ser estatisticamente significativo, nos indivíduos obesos, as médias da pontuação das dimensões da QV encontraram-se todas inferiores em relação aos não obesos (exceto no Desempenho Emocional), com maior destaque nas dimensões Dor e Vitalidade. Na análise da variável Doença crónica, verificou-se que esta influencia significativamente as dimensões Função Física, Estado de Saúde Geral e Vitalidade, sendo que os portadores de doença crónica apresentaram pontuações menores em todas as dimensões (com exceção da dimensão Desempenho Físico). No 
que diz respeito à variável Baixa médica, verificou-se que esta apresentou diferenças estatisticamente significativas nas dimensões Função Social $(62,5$ vs. 81,8) e Saúde Mental $(43,0$ vs. 71,9$)$, sendo que os que estiveram de Baixa médica apresentaram médias de pontuações da QV menores, o que também se verificou nas restantes dimensões, exceto na dimensão Desempenho Físico.

Relativamente às condições de trabalho, apenas se verificaram diferenças significativas nas variáveis Função de Direção e a dimensão Desempenho Físico, sendo que os trabalhadores de escritório que exercem Funções de Direção apresentaram menores pontuações comparativamente aos que não exercem essas funções (38,5 vs. 65,9, respetivamente); na variável Situação atual de emprego não se verificaram diferenças estatisticamente significativas.

Quanto às variáveis que foram analisadas através de cálculos de regressão linear, mostraram-se estatisticamente significativas a Idade Categorizada com a dimensão Função Social e Horas Extraordinárias com as dimensões Desempenho Físico e Função Social, contudo apresentaram correlação muito fraca, pelo que não foram consideradas relevantes para o presente estudo.

\section{DISCUSSÃO}

Pretendeu-se com este estudo avaliar a perceção da QV dos trabalhadores de escritório. Desse modo, os participantes que constituem a amostra apresentaram globalmente boa perceção da QV sendo as dimensões físicas as que de modo geral mais contribuem para essa qualidade com exceção do Desempenho Físico. As dimensões mentais são as que se apresentaram mais afetadas, nomeadamente o Desempenho Emocional e a Vitalidade, refletindo-se na quantidade de trabalho e ainda dos níveis de energia e de fadiga. Importante referir que algumas dimensões da QV apresentaram valores elevados de desvio padrão, nomeadamente o Desempenho Emocional e o Desempenho Físico. Essa discrepância pode ser explicada pela individualidade de cada trabalhador e pelos fatores extralaborais, que influenciam a perceção da QV dos trabalhadores de escritório, apesar das condições de trabalho serem semelhantes para todos. No entanto, em todas as dimensões da QV, a amostra em estudo obteve médias superiores a 50 , pelo que se considera que, no presente estudo, os trabalhadores de escritório apresentaram valores de perceção da QV muito bons (26). Um estudo a 35 funcionários técnico administrativos (média de idade de 42,0 anos $\pm 13,20$ ) realizado por Vey at al., (27), apresentou resultados semelhantes, no que diz respeito ao domínio da Vitalidade (pontuação média de 61,0). Segundo a Organização Internacional do Trabalho (OIT) (28), estes resultados, em trabalhadores de escritório, podem estar relacionados com o facto de utilizarem permanentemente (sempre na mesma posição) computadores durante o dia, que provoca uma sobrecarga do sistema neuro-musculoesquelético, e consequentemente fadiga a esse nível. 
Relativamente às variáveis sociodemográficas, existem diferenças entre géneros em todas as dimensões da QV, verificando-se que as pontuações do género masculino são superiores, exceto na dimensão Estado de Saúde Geral. No entanto, essas diferenças são estatisticamente significativas apenas nas dimensões Vitalidade e Dor, ou seja, o género masculino apresentou uma melhor perceção da QV. Estes dados vão de encontro a um estudo transversal que pretendia avaliar a perceção da QV em 158 funcionários do Instituto Politécnico do Porto (média de idade 41,7 anos $\pm 8,80$ ), que referiu valores inferiores no género feminino, justificados pela maior propensão das mulheres para relatar a doença e, consequentemente, referir desconforto/ dor, interferindo negativamente com o seu desempenho no dia-a-dia (29). Também, segundo a literatura, é importante referir que a população do género feminino passa habitualmente um número de horas consideravelmente maior, envolvidas após o horário laboral, em tarefas domésticas e apoio familiar, o que pode justificar menores níveis de energia e maior fadiga, ou seja, menor média de pontuação na dimensão da QV, Vitalidade (28).

No que diz respeito às condições de saúde, ao analisar os dados do IMC agrupados em obesos e não obesos, concluiu-se que a população obesa apresentou pontuações inferiores à não obesa nas diferentes dimensões da QV, exceto no Desempenho Emocional e, assim sendo, tanto as dimensões físicas como mentais se encontraram afetadas. No estudo realizado por Molero et al., (30) com uma amostra de 10033 participantes verificou-se o impacto negativo da obesidade na $Q V$, nomeadamente nas dimensões físicas, o que pode ser explicado pela maior sintomatologia dolorosa e pior performance física na população com IMC mais elevado (30). Por outro lado, a autoestima dos indivíduos obesos encontra-se por vezes diminuída devido à pressão social, o que tal como demonstra o estudo de Molero (30), pode levar a níveis mais elevados de ansiedade e depressão, influenciando negativamente a perceção da QV nas dimensões mentais (31). Contudo, é de salientar que a única dimensão estatisticamente significativa e relevante é o Estado de Saúde Geral, pelo que os trabalhadores de escritório obesos têm uma perceção de saúde e do seu aspeto mais negativo.

Relativamente aos portadores de Doença Crónica, a análise estatística revelou que estes apresentaram pontuações significativamente inferiores nas dimensões Estado de Saúde Geral, Vitalidade e Função Física. No entanto, os indivíduos portadores de Doença Crónica apresentaram valores elevados na dimensão Função Física, pelo que não se pode considerar que esta influencia negativamente a QV. O estudo de Marcarine et al., (32), efetuado numa amostra com 579 indivíduos e com uma média de idade de 42,7 anos $\pm 13,74$ evidenciou que os indivíduos com maior número de lesões ou doenças apresentaram resultados significativamente menores em todas as dimensões da QV. Também, Mascarenhas et al., (33) verificou que a dor decorrente de lesões interfere na $\mathrm{QV}$, em específico nas dimensões físicas, pois a sintomatologia dolorosa pode comprometer as atividades da vida diária e consequentemente a vida profissional. Neste estudo verificou-se ainda que a média da pontuação da dimensão Desempenho Emocional se encontrava relativamente baixa e, de facto, a Doença Crónica também resulta em perturbações emocionais que conduzem à alteração do funcionamento e rotina diária, interferindo com a vida profissional, familiar e social, e, inevitavelmente, com a QV (4). 
A presença de lesões ou doenças pode originar diversos desconfortos bem como ausências no trabalho. No sentido de perceber como essas ausências interferem com a QV, foi analisada a relação entre a Baixa médica por motivos de doença ou acidente, no último ano, com as dimensões em estudo. Neste estudo, as dimensões mentais destacaram-se como sendo negativamente mais influenciadas pela Baixa Médica, comparativamente com as dimensões físicas. Tal pode ser explicado pelo impacto dos problemas físicos ou mentais na socialização, por problemas como ansiedade, depressão ou sensação de mal-estar psicológico que possam estar associados ao motivo da Baixa. Segundo a Direção Geral da Saúde (2), as limitações funcionais apresentam um elevado custo e impacto na QV de cada trabalhador gerando consequências para as pessoas, para as empresas que sofrem níveis mais elevados de absentismo por doença e quedas na produtividade, e para a sociedade em geral (custos para o sistema de saúde) $(18,34)$.

Em relação às condições de trabalho, quando relacionado o número de Horas extraordinárias com as dimensões da QV, verificou-se que a correlação das variáveis não é válida para o estudo em questão devido às médias das pontuações nas dimensões da perceção da QV serem muito dispersas. Apesar disso, verificou-se que, em todas as dimensões, os trabalhadores de escritório que realizam mais horas de trabalho têm pontuações médias inferiores, pelo que, apesar das diferenças não serem significativas, existe alguma influência desta variável na perceção da QV dos trabalhadores de escritório, principalmente na dimensão Desempenho Emocional. A OIT (28), refere que $36,1 \%$ dos trabalhadores mundiais cumprem horários de trabalho excessivos. Essas horas extraordinárias em trabalhadores de escritório, traduzem-se para mais tempo prolongado no conjunto postural de sentado, o que, segundo a Agência Europeia para a Segurança e a Saúde no Trabalho (20), aumenta o risco de desenvolver doenças crónicas e queixas neuromusculoesqueléticas. Decorrente disso, a fadiga e o stress pode interferir com a saúde mental pelo aumento dos níveis de ansiedade, depressões e distúrbios do sono $(19,28)$.

Da análise aos trabalhadores de escritório que exercem Funções de Direção, verificou-se que apresentaram pontuações mais baixas em todas as dimensões, no entanto apenas foram significativas na dimensão Desempenho Físico. De facto, como demonstra Beneli (13), neste setor de trabalho, a alta carga mental, o alto grau de responsabilidade e a intensa exposição ao computador são fatores que influenciam a QV e, mais especificamente, a capacidade em desempenhar as suas funções devido a limitações, tanto físicas como mentais $(12,19)$.

Por último, na variável Situação atual de emprego não foram encontrados valores estatisticamente significativos, no entanto, um trabalho temporário ou a tempo parcial pode provocar stress constante nos trabalhadores devido a um sentimento de insegurança profissional que por sua vez pode atuar na perceção da QV, apesar desse impacto não ser marcante no presente estudo (28).

A avaliação da QV é fundamental para medir problemas que interferem no bem-estar e na vida dos indivíduos (9). Como forma de aumentar o desempenho geral e a promoção de saúde, as empresas apostam cada vez mais no design ergonómico dos postos de 
trabalho e na implementação de pausas ativas $(2,6,10,12,13,17)$. Ofertas implementadas, nomeadamente pausas ativas, classes de Pilates e alterações da ergonomia, na empresa de onde provém a amostra, podem ter influenciado positivamente os resultados do estudo e justificar maiores pontuações da QV nas dimensões físicas. Também é de referir que os trabalhadores de escritório do presente estudo trabalham num espaço aberto, com bastante luz natural e sem divisões, o que promove maior interação e menor monotonia entre eles. Tudo isto contribui para repor energias, diminuir o desconforto durante o trabalho, promover a socialização, diminuir o sedentarismo e o stress, influenciando positivamente todas as dimensões da QV avaliadas, o que pode explicar o facto da perceção da QV nos trabalhadores de escritório ser globalmente boa.

Foram encontradas como limitações do estudo o questionário apresentar uma questão relativa ao estado geral de saúde há um ano atrás, e sete questões acerca da perceção de saúde nas várias dimensões há quatro semanas atrás, esta distância temporal pode levar a um viés de memória.

É importante referir que a amostra foi constituída por participantes voluntários, critério de seleção que constitui um viés de participação, dado que, à partida, são os trabalhadores mais proativos e mais interessados na sua QV os respondentes. No entanto, este estudo foi realizado sobre uma amostra representativa da população, com $n=119$, através do cálculo amostral observou-se que a margem de erro era inferior a 10\%, o que é estatisticamente aceitável (35).

Para estudos futuros, recomendamos que no questionário seja feita a questão relativamente ao número de anos de serviço, que é uma questão pertinente a ter em conta, e que influencia muitas vezes a QV dos trabalhadores, como defende Barbosa (29). Também, causas multifatoriais, que não foram questionadas, podem influenciar a perceção da QV, tal como como a qualidade do sono, o consumo de tabaco e de bebidas alcoólicas ou ainda as relações interpessoais (36).

\section{CONCLUSÃO}

Com este estudo concluiu-se que os trabalhadores de escritório apresentaram globalmente uma boa perceção da QV nas diferentes dimensões avaliadas. Os resultados mostraram que as dimensões Desempenho Emocional e Desempenho Físico foram as que apresentaram menores pontuações, portanto diminuíram a perceção de QV dos CW da amostra e, pelo contrário, a dimensão Função Física foi a que apresentou maior pontuação.

Quanto às variáveis sociodemográficas verificou-se que o género feminino apresenta menos perceção de QV nas dimensões Dor e Vitalidade, comparativamente ao género masculino. A variável Obesidade (ser obeso) influenciou negativamente o Estado de Saúde Geral. Nas restantes variáveis associadas às condições de saúde, verificou-se 
que os portadores de doença crónica e os que estiveram de baixa médica apresentaram médias das pontuações notavelmente inferiores, principalmente nas dimensões mentais. Quanto às variáveis relativas às condições de trabalho, concluiu-se que as altas cargas de trabalho (exercer horas extraordinárias e/ou funções de direção) são um fator negativo a ter em conta na perceção da QV dos trabalhadores de escritório desta amostra.

Este estudo permitiu entender quais as dimensões mais afetadas na perceção da QV dos trabalhadores de escritório, e assim direcionar a intervenção da equipa de saúde ocupacional no sentido de promover uma melhor perceção de QV na população alvo.

As medidas implementadas na empresa em estudo potenciaram a boa perceção da QV dos seus trabalhadores, pelo que servirão como modelo a propor em empresas semelhantes.

\section{CONFLITOS DE INTERESSE}

Os autores declaram não haver conflitos de interesse.

\section{AGRADECIMENTOS}

Agradecemos à Professora Doutora Cristina Melo pelo apoio prestado no decorrer da realização do presente estudo e aos trabalhadores e empresa, pela participação e disponibilidade prestada.

\section{Referências bibliográficas}

1. Organização Mundial da Saúde. WHOQOL: Measuring Quality of Life [Internet]. World Health Survey. 2020 [cited 2020 May 2]. Available from: https://www.who.int/healthinfo/survey/whoqol-qualityoflife/en/

2. Direção Geral da Saúde. Programa Nacional de Saúde Ocupacional (PNSOC). Lisboa; 2018.

3. Mello J, De Jesus R, Mello A. As dimensões da qualidade de vida no trabalho expressas nas diretrizes organizacionais: um estudo sob a perspectiva de Walton. Rev Bras Qual Vida. 2015;7(1).

4. Canavarro M, Pereira M, Moreira H, Paredes T. Qualidade de Vida e Saúde: Aplicações do WHOQOL. Alicerces. 2010;III(3). 
5. Lacaz F. Qualidade de vida no trabalho e saúde do trabalhador: uma visão crítica. In: O Clássico e o Novo. 2003.

6. Delani D, Pinho S. Labor Gymnastics: Improving the Quality of Life of Worker. Rev Científica da Fac Educ e Meio Ambient. 2013;4(1).

7. Mendes S. Avaliação do estado subjectivo de saúde: utilização de questões singulares por diferentes modos de administração [Internet]. Universidade do Porto, Faculdade de Medicina, Instituto de Ciências Biomédicas Abel Salazar; 2009. Available from: http://repositorio-aberto.up.pt/handle/10216/21908

8. Carvalho F. Condições de trabalho e saúde: diagnosticar para intervir. 2011.

9. Praça M. Qualidade de vida relacionada com a saúde: a perspectiva dos utentes que frequentam os Centros de Saúde do ACES Trás-os- Montes I Nordeste. 2012.

10. Lanhers C, Pereira B, Garde G, Maublant C, Dutheil F, Coudeyre E. Evaluation of 'IPreventive': a digital preventive tool for musculoskeletal disorders in computer workers - a pilot cluster randomised trial. BMJ Open. 2016;6(9).

11. Oliveira D. O Efeito de um programa de Atividade Física específico aplicado em operários fabris. 2017.

12. Gunendi G, Gungor O, Alptekin H, Alptekin J. The effect of Ergonomics Arrangemnets Accompanying Posture Exercises for Office Workers on pain and Quality of life. RJLBPCS. 2018;4(3).

13. Beneli L. Efeitos de um programa de ginástica laboral sobre a incidência de dor em funcionários de uma empresa de software. Rev Saúde e Meio Ambient. 2017;4.

14. De Carvalho J. Avaliação da Eficacia dos Planos de Proteção aplicados a operadores com Lesões Musculo-Esqueléticas ao nivel do membro superior. Universidade de Lisboa, Faculdade de Motricidade Humana; 2016.

15. Figueira B. Associação dos Factores Ocupacionais com a Prevalência de Lesões Musculo-Esqueléticas Relacionadas com o Trabalho numa Fabrica de Industria Automovel. 2011;

16. Santos S. Avaliação ergonómica de postos de trabalho com aplicação de diferentes técnicas. 2019.

17. Ahmed I, Shaukat M. Computer users' ergonomics and quality of life-evidence from a developing country. Int J Inj Contr Saf Promot [Internet]. 2018;25(2). Available from: https://doi.org/10.1080/17457300.2017.1415361

18. European Agency for Safety and Health at Work. Work-related musculoskeletal disorders: prevalence, costs and demographics in the EU. 2019.

19. Ferreira M, Shimano S, Fonseca M. Fisioterapia na avaliação e prevenção de riscos ergonômicos em trabalhadores de um setor financeiro. Fisioter e Pesqui. 2009;16(3).

20. European Agency for Safety and Health at Work. Work-related musculoskeletal disorders : prevalence, costs and demographics in the EU. 2019.

21. Organização Mundial da Saúde. Ambientes de trabalho saudáveis: um modelo para ação. 2010.

22. Ferreira P, Ferreira L, Pereira L. Medidas sumário física e mental de estado de saúde para a população portuguesa. Rev Port Saúde Pública [Internet]. 2012;30(2):. Available from: http://dx.doi.org/10.1016/j.rpsp.2012.12.007 
23. Severo M, Santos A, Lopes C, Barros H. Fiabilidade e validade dos conceitos teóricos das dimensões de saúde física e mental da versão Portuguesa do MOS SF-36. Acta Med Port. 2006;19(4).

24. Rand Health Care. 36-Item Short Form Survey (SF-36) Scoring Instructions [Internet]. 2020 [cited 2020 Jun 9]. Available from: https://www.rand.org/healthcare/surveys_tools/mos/36-item-short-form/scoring.html

25. Miot H. Correlation analysis in clinical and experimental studies. J Vasc Bras [Internet]. 2018;17(4). Available from: https://doi.org/10.1590/1677-5449.174118

26. Dos Santos B, Carvalho M, Rolemberg A, Fernandes M. Análise da qualidade de vida e acometimentos osteoarticulares relacionados às atividades laborais de funcionários públicos. 2016.

27. Vey A, Daronco L, Silva A, Souza L, Braz M, Temp H, et al. Qualidade de vida e satisfação no trabalho de funcionários técnico administrativos da Universidade Federal de Santa Maria. St Maria. 2015;41(1).

28. Organização Internacional do Trabalho. Segurança e Saúde no Centro do Futuro do Trabalho [Internet]. 2019. Available from: dgs.pt/saude-ocupacional/documentosso/relatorio-oit-abril-2019-pt-pdf.aspx

29. Barbosa V. A satisfação no trabalho e a perceção da qualidade de vida nos colaboradores do IPP. 2014.

30. Molero M, Villegas A, González M, Rastrollo M, Urbistondo M, Santabárbara J, et al. The influence of obesity and weight gain on quality of life according to the SF-36 for individuals of the dynamic follow-up cohort of the University of Navarra. Rev Clin Esp [Internet]. 2018;218(8). Available from: dgs.pt/saude-ocupacional/documentosso/relatorio-oit-abril-2019-pt-pdf.aspx

31. Jesus D, Santana M, Sousa R, Santana O, Boas A, Luz J, et al. Aptidão física e saúde: Educação Física, Saúde e Obesidade [Internet]. Report of births, marriages, and deaths in Massachusetts. . 2019. Available from: https://books.google.pt/books?id=VszO_kZl4bUC\&printsec=frontcover\&hl=pt-PT

32. Marcarine P, Castro S, Castro S, Meirelles M, Haas V, Walsh I. Quality of life, sociodemographic and occupational factors of working women. Cien Saude Colet. 2019;24(7).

33. Mascarenhas C, Prado F, Fernandes M. Factors associated with the quality of life of community health agents. Cien Saude Colet. 2013;18(5).

34. Sardinha J. Determinação de padrões de exposição ocupacional associados à prevalência de lesões músculo-esqueléticas numa indústria metalúrgica. 2018.

35. Feijoo A. A pesquisa e a estatística na psicologia e na educação. 2010.

36. Talhada L. Qualidade do sono, saúde e estilos de vida Estudo com a população activa portuguesa. Universidade da Beira Interior; 2012.

Figura 1 - Fluxograma da amostra 


\section{(1)Sara Moreira}

ICBAS, Instituto de Ciências Biomédicas Abel Salazar - Universidade do Porto. Escola Superior de Saúde - Instituto Politécnico de Viana do Castelo, Portugal. CBSin, Centro de investigação de Biociências em Saúde Integrativa, Porto, Portugal. Morada para correspondência dos leitores: Rua de Jorge Viterbo Ferreira, 228, 4050-313 Porto, Portugal. E-mail: sama@ess.ipvc.pt. ORCID iD: https://orcid.org/0000-0003-1585-1142

\section{(2)Ana Miranda}

ESS|PPorto - Escola Superior de Saúde - Instituto Politécnico do Porto, Portugal. Clínica Neuro Studium, Boavista - Porto, Portugal. 4415-629, Vila Nova de Gaia. E-mail: anafsmiranda.98@gmail.com

\section{(3)Carolina Lima}

ESS|PPorto - Escola Superior de Saúde - Instituto Politécnico do Porto, Portugal. FisioGaia - Clínica de Recuperação Funcional, Vila Nova de Gaia, Porto, Portugal. 4905612 Geraz do Lima (Sta Maria), Viana do Castelo. E-mail: carolina.rodrigueslima@hotmail.com

\section{(4)Cristina Gonçalves}

ESS|PPorto - Escola Superior de Saúde - Instituto Politécnico do Porto, Portugal. Santa Casa da Misericórdia São Miguel de Refojos, Cabeceiras de Basto - Braga. 4850-477 Vieira do Minho. E-mail: cristinagoncalves_7@hotmail.com

\section{(5)Cristina Mesquita}

ESS|PPorto - Departamento de Fisioterapia, Escola Superior de Saúde, Politécnico do Porto, Portugal. CIR - Centro de Investigação e Reabilitação, ESS|PPorto, Portugal. 4200-072 Porto. E-mail: ctmesquita@ess.ipp.pt. ORCID iD: https://orcid.org/0000-00030830-9064

\section{(6)Sofia Lopes}

ESS|PPorto - Departamento de Fisioterapia, Escola Superior de Saúde, Politécnico do Porto, Portugal. CIR - Centro de Investigação e Reabilitação, ESS|PPorto, Portugal. Departamento de Tecnologias de Diagnóstico e Terapêutica, Escola Superior de Saúde do Vale do Sousa, Instituto Politécnico de Saúde do Norte (IPSN), CESPU, Gandra, Portugal. 4200 - 072, Porto. E-mail: sofiarochalopes@gmail.com. ORCID iD: https://orcid.org/0000-0002-3306-7557

\section{(7)Jorge Machado}


ICBAS, Instituto de Ciências Biomédicas Abel Salazar - Universidade do Porto. CBSin, Centro de investigação de Biociências em Saúde Integrativa, Porto, Portugal. CIIMAR, Centro Interdisciplinar de Pesquisa Marinha e Ambiental, Universidade do Porto, Portugal. 4050-313 Porto, Portugal. E-mail: jmachado@icbas.up.pt. ORCID iD: http://orcid.org/0000-0003-4570-5445

\section{(8)Paula Clara Santos}

ESS|PPorto - Departamento de Fisioterapia, Escola Superior de Saúde, Politécnico do Porto, Portugal. CIAFEL-Centro de Investigação em Atividade Física, Saúde e Lazer, Faculdade de Desporto, Universidade do Porto, Portugal. CIR - Centro de Investigação e Reabilitação, Escola Superior de Saúde, Politécnico do Porto, Portugal. 4200 - 072 , Porto. E-mail: paulaclara.santos3@gmail.com. ORCID iD: https://orcid.org/0000-00033548-700X 